\title{
Birth Weight in Relation to Post-Natal Growth Patterns as Predictor of Arterial Stiffness and Central Hemodynamics in Young Adults from a Population-based Study
}

\author{
Johannes Sperling, Shantanu Sharma (D), Peter M. Nilsson**iD \\ Department of Clinical Sciences, Lund University, Skane University Hospital, Internal Medicine Research Group, Jan Waldenströms gata 15, \\ Floor 5, Malmö S-20502, Sweden
}

\section{ARTICLE INFO}

Article History

Received 06 November 2020

Accepted 24 January 2021

Keywords

Augmentation index birth weight

central blood pressure

mismatch

pulse wave velocity

\begin{abstract}
Objective: Our aim was to examine the impact of mismatch patterns reflecting pre- and post-natal growth conditions on markers of arterial stiffness and central hemodynamics in young adults.

Methods: In all, 1056 participants from Malmö Offspring Study, 484 men and 572 women (age-range 18-44 years), were included. All participants were stratified into four subgroups based on low $(\leq 0)$ or high $(>0)$ Birth Weight $z$-score (BWz) and low ( $\leq$ median) or high (> median) Body Mass Index (BMI) at 20 years age (BMI20). All participants underwent carotidfemoral Pulse Wave Velocity (PWV) measurement and pulse wave analysis with Sphygmocor. Additionally, 24-h ambulatory blood pressure data was recorded in a subgroup of 184 participants.
\end{abstract}

Results: Systolic Blood Pressure (SBP), central SBP (cSBP) and Diastolic Blood Pressure (DBP), and 24-h night-time SBP was higher ( $p<0.001 ; p<0.001 ; p=0.04$ ) in "low BWz/high BMI20" (mismatch group) compared with "low BWz/low BMI20" (reference). The mismatch phenotype was significantly associated with an increased risk of elevated brachial [odds ratio (OR), 2.78; 95\% confidence interval (CI), 1.94-3.98] and cSBP (OR, 2.0; CI: 1.38-2.91) in young adults. No differences were observed in PWV or augmentation pressure index in comparison between "low BWz/high BMI20" and "low BWz/low BMI20."

Conclusion: Lower birth weight in combination with a higher attained BMI in young adult life, is associated with higher brachial SBP/DBP and central SBP/DBP. Therefore, children born with low birth weight should be protected from exaggerated catch-up growth to reduce their risk of adult hypertension, obesity, and adverse central hemodynamics.

\section{HIGHLIGHTS}

We aimed to examine the impact of mismatch patterns between pre- and post-natal growth conditions on markers of arterial stiffness and central hemodynamics in 1056 participants from a population-based study in Sweden, 484 men and 572 women in the age-range $18-44$ years.

- Lower birth weight was associated with higher Brachial DBP (bDBP), higher central SBP/DBP, and higher Aix.

- Lower birth weight in combination with a higher attained BMI in young adult life (the mismatch phenotype) associates with higher bSBP/bDBP and higher central blood pressure.

- We suggest an additive hemodynamic programming effect of weight gain during the two first decades of life following low birth weight.

(c) 2021 The Authors. Publishing services by Atlantis Press International B.V. This is an open access article distributed under the CC BY-NC 4.0 license (http://creativecommons.org/licenses/by-nc/4.0/).

\section{INTRODUCTION}

Low birth weight, adjusted for gestational age, has repeatedly been associated with an increased risk of cardiovascular mortality, hypertension, coronary heart disease, and stroke [1-5]. The mechanisms behind these associations are not yet fully understood. While low birth weight for gestational age is considered a phenotype influenced by fetal growth restriction, also small changes

Corresponding author.Email: Peter.Nilsson@med.lu.se

Peer review under responsibility of the Association for Research into Arterial Structure and Physiology

Data availability statement: Data are available upon reasonable request and application sent to Professor Olle Melander, Chair of the Board for the Malmö Offspring Study, as part of the Malmö Population-based Cohorts (https://www.malmo-kohorter.lu.se/english) in the fetal or prenatal environment may cause fetal adaptions through epigenetic reprogramming. These new insights have led to a shift from the original Barker's hypothesis [6], featuring fetal growth restriction in utero as a response to the maternal environment, toward the concept of developmental origins of health and disease. This suggests that early environmental challenges affect an individual's later risk of organ dysfunction and disease through impaired growth and epigenetic changes, so-called fetal programming [7]. The fetal response and adaption to early life factors may be beneficial in the womb but disadvantageous outside, causing a mismatch between pre- and post-natal environments. For instance, poor nutrition in fetal life expressed as lower birth weight, followed by adequate or rich nutrition during the early years puts the baby at a higher risk of rapid catch-up growth, so called 
Accelerated Post-natal Growth (APG). The growth pattern in APG seems different from normal post-natal growth, with weight increasing at a higher velocity than length, at least during the first 2 years $[8,9]$. APG has been proposed as a more important risk marker for future Cardiovascular Disease (CVD) than low birth weight or body size alone [10,11]. Also, APG following fetal growth restriction is associated with an increased risk of obesity/ overweight, higher levels of insulin and blood pressure, as well as higher Carotid-Femoral Pulse Wave Velocity (cf-PWV) during both childhood and in early adulthood [12-15]. Comparatively, in adults, a history of fetal growth restriction is associated with an increased risk of obesity, type 2-diabetes and CVD [16,17]. Furthermore, in adults with a history of low birth weight followed by APG, higher Systolic Blood Pressure (SBP) and Diastolic Blood Pressure (DBP) are observed $[18,19]$. This association may be further strengthened by a higher attained adult Body Mass Index (BMI) [20,21].

The present study aimed to examine the mismatch between preand post-natal factors influencing adult body weight for the prediction of central and peripheral hemodynamics in a population-based cohort of young adults.

\section{SUBJECTS AND METHODS}

\subsection{Study Population}

The study design was observational and cross-sectional with the inclusion of 1056 participants from Malmö Offspring Study (MOS) [22,23], a total of 484 men and 572 women in the age-range 18-44 (mean 28.6) years. Selection criteria were: (a) participants who underwent cf-PWV measurement, (b) with available birth characteristic data, and (c) a recalled body weight at 20 years (for those 20 years or older). Because not all participants in MOS underwent ambulatory 24-h blood pressure measurements, such data were only available for a total of 184 participants during the first years of MOS. Similarly, Pulse Wave Analysis (PWA) was only performed in 1010 participants to evaluate central blood pressure and the Augmentation Index (Aix).

Malmö Offspring Study is an ongoing cohort study that started in 2013 at the Skåne University Hospital, Malmö, Sweden. The study consists of children and grandchildren to subjects from the cardiovascular arm of the Malmö Diet and Cancer Study [24]. In addition, birth weight and gestational age data were derived from the Swedish Medical Birth Register (MBR), a national register collecting birth characteristic data from obstetrics clinics of all registered newborns since 1973, previously used in MOS [22].

The Regional Ethical Board at the Lund University, Sweden, provided ethical permission (Dnr. 2012/594), and all participants gave their written informed consent.

\subsection{Clinical Examinations}

All participants underwent a physical examination on the same day as cf-PWV measurement including, height $(\mathrm{cm})$ and weight $(\mathrm{kg})$ in light indoor clothes. Office SBP and DBP were measured (mmHg) with an automatic device (OMRON M5-1 IntelliSense, Brighton, UK) after $5 \mathrm{~min}$ of rest in the supine position, and a mean was calculated from three repeated measurements.
The cf-PWV was measured with Sphygmocor XCEL (Atcor, Australia), using applanation tonometry with a high-fidelity sensor. The pulse wave was recorded at the site for carotid and femoral artery pulsation to calculate foot-to-foot time between the pulse waves. Furthermore, the distance was manually measured from the site of carotid artery pulsation to the suprasternal notch, from suprasternal notch to umbilicus, and from the umbilicus to the site of femoral artery pulsation [25]. Then PWV was calculated by the manufacturer's software. Likewise, pulse wave was recorded at the site of radial artery pulsation with a high-fidelity sensor using applanation tonometry. Thereafter, a central pressure waveform was estimated with a transfer function, also calculating the central aortic pressure and central SBP (cSBP) and central DBP (cDBP). Moreover, Aix was defined as the ratio of the difference between the early and late systolic peak and early systolic peak, or the difference between early and late systolic peak divided by Pulse Pressure (PP).

Before the examination, all participants were asked to abstain from alcohol for $12 \mathrm{~h}$ and nicotine or heavy meals for at least $4 \mathrm{~h}$. Furthermore, all procedures were performed by trained staff following strict protocols in a room with regulated temperature and dimmed light, with the participant in a supine position resting for 5 min before start.

In a subgroup of 184 participants, 24-h Ambulatory Blood Pressure Monitoring (ABPM) was performed using $24 \mathrm{~h}$ Arteriograph (Tensiomed, Budapest, Hungary). Daytime and night-time mean for SBP, DBP, and CSBP were used in the present study. This examination was used only during the first years of MOS and subjects were not selected specifically.

\subsection{Statistical Analysis}

Data are presented as mean \pm Standard Deviation (SD) for all continuous variables, otherwise as medians with interquartile range (first and third quartile). Also, extreme outliers were carefully reviewed and excluded if outside physiological ranges or obviously wrongly reported. All statistical analyses were performed with SPSS version 25.0 (IBM, Armonk, NY, USA). For general characteristics, comparison of means between men and women was performed with independent $t$-test for parametric data and Mann-Whitney $U$-test for non-parametric data. A $p<0.05$ was considered statistically significant in all analyses.

Birth weight was converted to $z$-score adjusted for gestational age (in days) and sex, based on equations calculated from official Swedish growth-charts [26]. Furthermore, BMI was calculated as weight divided with height for the square meter $\left(\mathrm{kg} / \mathrm{m}^{2}\right)$. A variable was defined by BMI-calculation for recalled weight at 20 years of age (BMI20), reported in a questionnaire, but with actual height. For those participants aged 20 years or younger, their actual body weight was used instead.

To test the mismatch hypothesis, all participants were divided into four subgroups based on low $(\leq 0)$ or high $(>0)$ Birth Weight $z$-scores (BWz), and low ( $\leq$ median) or high ( $>$ median) BMI20. Hence, four subgroups were defined: (a) low BWz/Low BMI20 (reference), (b) low BWz/high BMI20 (the mismatch phenotype), (c) high BWz/low BMI20, and (d) high BWz/high BMI20, for all subjects. In addition, we stratified for gender by dividing men and women, respectively, into four subgroups based on the same method described above. 
The body weight at 20 years was preferred over actual weight at examination assuming a phenotype time-wise closer to the true phenotype of APG. Further, comparison between low BWz/ low BMI20 (reference) and other subgroups was performed with Analysis of Variance (ANOVA) for normally distributed data, or Kruskal-Wallis test for non-parametric data. A Bonferroni post hoc test was used in ANOVA and Bonferroni correction in KruskalWallis for post hoc testing.

The Odds Ratio (OR) with 95\% Confidence Interval (CI) was calculated [27], for Brachial SBP (bSBP), cSBP, and cf-PWV as well as Aix. For each variable, the 75th percentile in the reference group was used as a cutoff for high/low definitions. Calculations were made for all participants, and in men and women, separately. $p$-Values were calculated for significance testing.

Finally, multiple linear regression was performed using $\mathrm{BWz}$ as an independent variable and cf-PWV, Aix, bSBP/bDBP, cSBP/DBP as well as $24 \mathrm{~h}$ blood pressures as dependent variables, following adjustment for age and sex. In addition, cSBP was added as a covariate for cf-PWV and Aix.

\section{RESULTS}

\subsection{General Characteristics}

General characteristics for all participants and stratified for gender are presented in Table 1.

\subsection{Comparison between Subgroups}

A comparison between the four mismatch subgroups defined by BWz and BMI20 for all participants is presented in Table 2.

Office SBP was higher $(p<0.001)$ in "low BWz/high BMI20" (mismatch) and "high BWz/high BMI20" compared with "low BWz/ low BMI20" (reference). Furthermore, cSBP was higher $(p<0.001$, $p=0.05$ ) in "low BWz/high BMI20" (mismatch) and at borderline significance in "high BWz/high BMI20", but lower $(p=0.01)$ in "high BWz/low BMI20", compared with reference.

Office DBP was higher $(p=0.049)$ in "low BWz/high BMI20" (mismatch) compared with reference. Office DBP and cDBP were lower $(p=0.015 ; p<0.001)$ in "high BWz/low BMI20" compared with reference.

Augmentation index was lower $(p<0.001 ; p=0.008)$ in "high BWz/ low BMI20" and "high BWz/high BMI20", both compared with the reference group after adjustment for adult height.

For 24-h ABPM, mean daytime SBP was higher $(p=0.011)$ in "high BWz/high BMI20" compared with reference. Furthermore, mean night-time SBP was higher $(p=0.04)$ in "low BWz/high BMI20" (mismatch) and "high BWz/high BMI20" ( $p=0.001)$ compared with reference.

Daytime pulse pressure was higher in "high BWz/high BMI20" $(p=0.019)$ compared to the reference group. Finally, night-time pulse pressure was higher $(p=0.026)$ in "low BWz/high BMI20" (mismatch) and "high BWz/high BMI20" ( $p=0.011)$ compared with reference.

Table 1 Characteristics of the total study population and stratified for gender

\begin{tabular}{lccc}
\hline Variables & All & Men & Women \\
\hline General characteristics & $n=1056$ & $n=484$ & $n=572$ \\
Age (years) & $28.6(6.75)$ & $28.9(6.89)$ & $28.3(6.61)$ \\
Height (cm) & $174.4(9.65)$ & $182(7.17)^{*}$ & $167.9(6.1)$ \\
Weight (kg) & $74[64-86]$ & $75[70-84]^{*}$ & $66[59-75.5]$ \\
Weight at 20 years (kg) & $68[60-77.75]$ & $83[74.25-93]^{*}$ & $62[56-68]$ \\
BMI at 20 years age (kg/m $\left.{ }^{*}\right)$ & $22.3[20.57-24.42]$ & $23.1[21.14-24.82]^{*}$ & $21.7[20.03-23.67]$ \\
Birth characteristics & $n=1056$ & $n=484$ & $n=572$ \\
Birth weight (g) & $3472.1(600.75)$ & $3504.7(664.4)$ & $3444.5(540.14)$ \\
Birth weight $z$-score & $-0.1(1.17)$ & $-0.2(1.18)$ & $-0.1(1.57)$ \\
Hemodynamic parameters & $n=1056$ & $n=484$ & $n=572$ \\
SBP (mmHg) & $110.9(11.62)$ & $117[111-124]^{*}$ & $104.8(9.11)$ \\
DBP (mmHg) & $67.1(7.41)$ & $67[63-72]^{*}$ & $66[62-71]$ \\
Pulse wave velocity (m/s) & $6.5(0.98)$ & $6.7(1.02)^{*}$ & $6.3(0.9)$ \\
Pulse wave recordings & $n=1010$ & $n=466$ & $n=544$ \\
Augmentation pressure index & $-1.7(11.74)$ & $-5.1(10.65)^{*}$ & $1.1(11.89)$ \\
Central SBP (mmHg) & $94.3(9.3)$ & $98[93-103]^{*}$ & $90.7(8.36)$ \\
Central DBP (mmHg) & $67.3(7.35)$ & $67[63-72]$ & $66.9(7.05)$ \\
ABPM (mmHg) & $n=184$ & $n=80$ & $n=104$ \\
SBP daytime & $120.3(10.49)$ & $125.6(9.41)^{*}$ & $116.3(9.44)$ \\
SBP night-time & $105.3(10.44)$ & $109.6(9.54)^{*}$ & $102(9.9)$ \\
DBP daytime & $69.6(8.6)$ & $73.1(8.98)^{*}$ & $67(7.3)$ \\
DBP night-time & $57(8.15)$ & $59.8(8.41)^{*}$ & $54.9(7.31)$ \\
Central SBP daytime & $109[102-116]$ & $113.6(11.01)^{*}$ & $106.9(10.51)$ \\
Central SBP night-time & $96.3(10.42)$ & $98.9(9.66)^{*}$ & $94.3(10.6)$ \\
Pulse-pressure daytime & $50.7(5.38)$ & $39.1(16.19)^{*}$ & $39.9(5.35)$ \\
Pulse-pressure night-time & $48.3(5.74)$ & $49.8(5.89)^{*}$ & $47(5.33)$ \\
\hline Dapreseras & & & \\
\end{tabular}

Data presented as mean $( \pm \mathrm{SD})$ or median [first and third quartile]. $p<0.05$ was considered statistically significant. "Statistical significance between men and women. $n$, number of subjects. 
Table 2 General characteristics of four subgroups based on low/high birth weight and low/high BMI at age 20 years, including all participants. The mismatch category, reflecting post-natal catch-up growth (Low BWz/High BMI20) is indicated in bold italic

\begin{tabular}{|c|c|c|c|c|}
\hline Variables & $\begin{array}{c}\text { Low BWz/Low BMI20 } \\
\text { (reference) }\end{array}$ & Low BWz/High BMI20 & High BWz/Low BMI20 & High BWz/High BMI2C \\
\hline General & $n=294$ & $n=271$ & $n=233$ & $n=258$ \\
\hline Age (years) & $29(6.8)$ & $28.9(6.7)^{\dagger}$ & $28.2(6.6)^{*}$ & $28.1(6.9)^{\ddagger}$ \\
\hline Height $(\mathrm{cm})$ & $171.9(9.3)$ & $174.2(9.2)^{\dagger}$ & $175.2(9.7)^{*}$ & $176.7(9.9)^{\ddagger}$ \\
\hline Weight $(\mathrm{kg})$ & $66.6(12)$ & $83.1(14.6)^{\dagger}$ & $68.2(11.3)$ & $85(16)^{\ddagger}$ \\
\hline Weight at 20 years $(\mathrm{kg})$ & $60.1(8.1)$ & $76.7(12)^{\dagger}$ & $62.9(7.9)^{*}$ & $78(12)^{\ddagger}$ \\
\hline BMI at 20 years $\left(\mathrm{kg} / \mathrm{m}^{2}\right)$ & $20.3(1.4)$ & $25.3(3.3)^{\dagger}$ & $20.4(1.3)$ & $25.4(2.9)^{\ddagger}$ \\
\hline Birth weight $(\mathrm{g})$ & $3107.6(482.2)$ & $3157.2(494)$ & $3806.5(471.1)^{*}$ & $3916(451.4)^{\ddagger}$ \\
\hline Office blood pressure & $n=294$ & $n=271$ & $n=233$ & $n=258$ \\
\hline SBP (mmHg) & $108.8(11.1)$ & $114.8(11.3)^{\dagger}$ & $106.8(10.6)$ & $112.9(11.6)^{\ddagger}$ \\
\hline $\mathrm{DBP}(\mathrm{mmHg})$ & $66[57-73]$ & $68[64-72]^{\dagger}$ & $64[59.5-68.5]^{*}$ & $66.5[61.5-71.5]$ \\
\hline Pulse wave measurements & $n=280$ & $n=259$ & $n=224$ & $n=247$ \\
\hline Pulse wave velocity (m/s) & $6.4(1)$ & $6.5(1)$ & $6.5(1)$ & $6.5(1)$ \\
\hline Augmentation pressure index & $0.6(12.2)$ & $-1.9(11.5)$ & $-3.5(11.5)^{*}$ & $-2.6(11.3)^{\ddagger}$ \\
\hline Central SBP $(\mathrm{mmHg})$ & $93.6(9.6)$ & $97(9)^{\dagger}$ & $90.6(8)^{*}$ & $95.7(9.2)$ \\
\hline Central DBP (mmHg) & $67[60.5-73.5]$ & $68[63.5-72.5]$ & $66[61.5-70.5]^{*}$ & $71[65.5-76.5]$ \\
\hline $\mathrm{ABPM}(\mathrm{mmHg})$ & $n=48$ & $n=46$ & $n=44$ & $n=46$ \\
\hline SBP day & $118.3(11.3)$ & $121.8(10.2)$ & $115.8(7.6)$ & $125.4(10.2)^{\ddagger}$ \\
\hline SBP night & $101.9(10.9)$ & $107.5(9.8)^{\dagger}$ & $102(8.3)$ & $109.8(10.7)^{\ddagger}$ \\
\hline DBP day & $68.6(10.3)$ & $69.4(8.4)$ & $67.9(6.4)$ & $72.6(8.2)$ \\
\hline DBP night & $55.2(8.8)$ & $57.9(8)$ & $55.6(7)$ & $59.5(8.2)$ \\
\hline PP daytime & $49.7(5.5)$ & $52.4(4.5)$ & $47.9(4.5)$ & $52.8(5.4)^{\ddagger}$ \\
\hline PP night-time & $46[42-50]$ & $50[46.5-53.5]^{\dagger}$ & $46[43-49]$ & $49.5[47-52]^{*}$ \\
\hline
\end{tabular}

Data presented as mean $( \pm \mathrm{SD})$ or median [25th-75th percentile] for all participants was divided into four groups. Low BWz/low BMI20 (reference group): BWz $\leq 0$ and $\mathrm{BMI} \leq$ median. Low BWz/High BMI20: $\mathrm{BWz} \leq 0$ and BMI $>$ median. High BWz/Low BMI20: BWz $>0$ and BMI $\leq$ median. High BWz/High BMI20: $\mathrm{BWz}>0$ and BMI $>$ median. Comparisons between reference group and each subgroup, respectively. $p<0.05$ was considered statistically significant. 'Significant differences between low BWz/ low BMI20 and low BWz/high BMI20. "Significant differences between low BWz/low BMI20 and high BWz/low BMI20. "Significant differences between low BWz/low BMI20 and high BWz/high BMI20. $n$, number of subjects.

\subsection{Comparison between Subgroups for Men}

Comparison between subgroups in men are presented in Table 3. Office bSBP and bDBP was higher $(p=0.022 ; p=0.01)$ as well as cSBP and cDBP $(p=0.006 ; p=0.032)$ in "low BWz/high BMI20" (mismatch) compared with reference. Office bDBP $(p=0.038)$, cSBP $(p=0.006), \operatorname{cDBP}(p=0.001)$ and Aix $(p=0.008)$ were all lower in "high BWz/low BMI20" compared with reference.

\subsection{Comparison between Subgroups for Women}

The comparison between subgroups in women is presented in Table 4. Office SBP $(p=0.001)$ and DBP $(p=0.023), \operatorname{cSBP}(p=0.03)$ and cDBP $(p=0.043)$ were all higher in "low BWz/high BMI20" (mismatch) compared with reference.

\subsection{Risk of Elevated Brachial and Central SBP}

Odds ratio for elevated bSBP and cSBP are presented in Table 5. The odds of higher bSBP ( $\geq 75$ th percentile of SBP in the reference group) was significantly higher in mismatch "low BWz/high BMI20" (OR 2.78; 95\% CI: 1.94-3.98) and "high BWz/high BMI20" (OR 1.92; 95\% CI: 1.33-2.78), but significantly lower in "high BWz/ low BMI20" (OR 0.55; 95\% CI: 0.35-0.86), all compared to the reference group. Correspondingly, the odds of higher cSBP (over 75th percentile of cSBP in the reference group) was significantly higher in mismatch "low BWz/high BMI20" (OR 2.0; 95\% CI: 1.38-2.91) but significantly lower in "high BWz/low BMI20" (OR 0.38; 95\% CI: 0.23-0.64) compared with reference.

\subsection{Risk of Elevated Brachial and Central SBP Stratified for Gender}

Odds ratio for elevated bSBP and cSBP in men and women respectively, are presented in Table 5. The odds of higher bSBP (over 75th percentile of SBP in the reference group) was significantly higher in men for mismatch "low BWz/high BMI20" (OR 2.31; 95\% CI: 1.36-3.93), and in women for mismatch "low BWz/high BMI20" (OR: 1.87; 95\% CI: 1.12-3.13), as well as "high BWz/high BMI20" (OR 1.7; 95\% CI: 1.02-2.82) compared with reference.

Correspondingly, the odds of higher cSBP was significantly higher in men for mismatch "low BWz/high BMI20" (OR: 1.79; 95\% CI: 1.02-1.13) compared with reference.

\subsection{Multiple Linear Regressions}

Birth weight $z$-score was positively associated with cf-PWV $(\beta=0.084 ; p=0.004)$, adjusted for sex, age and BMI at examination, SBP, DBP and heart rate. Furthermore, BWz was inversely 
Table 3 General characteristics of four subgroups of men based on low/high birth weight and low/high BMI at age 20 years. The mismatch category is indicated in bold italic

\begin{tabular}{lcccc}
\hline Variables & $\begin{array}{c}\text { Low BWz/Low BMI20 } \\
\text { (reference) }\end{array}$ & Low BW /High BMI20 & High BWz/Low BMI20 & High BWz/High BMI20 \\
\hline General & $n=138$ & $n=131$ & $n=104$ & $n=111$ \\
Age (years) & $29.5(6.8)$ & $29.3(6.8)$ & $28.4(7.3)$ & $28.4(7.3)$ \\
Height (cm) & $180.4(7.1)$ & $179.6(6.9)$ & $183.7(6.6)^{*}$ & $183.7(6.6)^{\ddagger}$ \\
Weight (kg) & $76.2(10.5)$ & $89.5(14.3)^{\dagger}$ & $77.4(7.5)$ & $94(16.2)^{\ddagger}$ \\
Weight at 20 years (kg) & $67(6.8)$ & $80.8(11.3)^{\dagger}$ & $70.4(5.8)$ & $86(10.8)^{\dagger}$ \\
BMI at 20 years (kg/m $\left.{ }^{\dagger}\right)$ & $20.5(1.3)$ & $25(3.1)^{\dagger}$ & $20.5(1.2)$ & $25.4(2.6)^{\ddagger}$ \\
Birth weight (g) & $3157.5(568.6)$ & $4001.9(542.5)$ & $3864.6(510.7)^{*}$ & $4001.9(542.5)^{\ddagger}$ \\
Office blood pressure & $n=138$ & $n=131$ & $n=111$ \\
SBP (mmHg) & $117.2(9.7)$ & $120.7(9.6)^{\dagger}$ & $n=104$ & $130(10.1)$ \\
DBP (mmHg) & $66.5[62-71]$ & $69[64.5-73.5]^{\dagger}$ & $115.3(9.8)$ & $64[59.5-68.5]^{*}$ \\
Pulse wave measurements & $n=136$ & $n=130$ & $n=102$ & $n=111$ \\
Pulse wave velocity (m/s) & $6.7(1)$ & $6.6(1)$ & $6.7(1)$ & $6.7(1)$ \\
Augmentation pressure index & $-3.3(10.7)$ & $-4.3(10.6)$ & $-7.8(9.9)^{*}$ & $-5.4(11)$ \\
Central SBP (mmHg) & $97[91.5-102.5]$ & $100.5[95.5-105.5]^{\dagger}$ & $94[90-98]^{*}$ & $98[92-104]$ \\
Central DBP (mmHg) & $66[62-70]$ & $69[65-73]^{\dagger}$ & $65[62-68]^{*}$ & $68[62.5-73.5]$ \\
ABPM (mmHg) & $n=21$ & $n=18$ & $n=21$ & $n=20$ \\
SBP day & $124.4(10.1)$ & $125.4(10)$ & $122.9(6.1)$ & $130(8.3)$ \\
SBP night & $108.1(10.5)$ & $108.6(8.2)$ & $107.7(5.3)$ & $114.3(9.6)$ \\
DBP day & $72.1(11)$ & $72.6(10.2)$ & $73.1(5.9)$ & $74.5(8.1)$ \\
DBP night & $59.2(9.7)$ & $59.2(8.9)$ & $59.4(5.8)$ & $61.4(9.2)$ \\
PP day & $52.3(5.9)$ & $52.8(3.9)$ & $49.8(3.9)$ & $55.5(5.5)$ \\
PP night & $48[43-53]$ & $49[46.5-51.5]$ & $48.5[47-50]$ & $51[46.5-55.5]$ \\
\hline
\end{tabular}

Data presented as mean $( \pm \mathrm{SD})$ or median [25th-75th percentile] for men was divided into four groups. Low BWz/Low BMI20 (reference group): BWz $\leq 0$ and BMI $\leq$ median. Low BWz/High BMI20: BWz $\leq 0$ and BMI $>$ median. High BWz/Low BMI20: BWz $>0$ and BMI $\leq$ median. High BWz/High BMI20: $\mathrm{BWz}>0$ and BMI $>$ median. Comparisons between reference group and each subgroup respectively. $p<0.05$ was considered statistically significant. ${ }^{\dagger}$ Significant differences between low BWz/low BMI20 and low BWz/high BMI20. "Significant differences between low BWz/low BMI20 and high BWz/low BMI20. ${ }^{\ddagger}$ Significant differences between low BWz/low BMI20 and high BWz/high BMI20. $n$, number of subjects.

Table 4 General characteristics of four subgroups of women based on low/high birth weight and low/high BMI at age 20 years. The mismatch category is indicated in bold italic

\begin{tabular}{lcccc}
\hline Variables & $\begin{array}{c}\text { Low BWz/Low BMI20 } \\
\text { (reference) }\end{array}$ & Low BWz/High BMI20 & High BWz/Low BMI20 & High BWz/High BMI20 \\
\hline General & $n=160$ & $n=136$ & $n=128$ & $n=148$ \\
Age (years) & $28.7(6.8)$ & $28.3(6.4)$ & $28.3(6.6)$ & $27.9(6.7)$ \\
Height (cm) & $167(5.9)$ & $166(5.8)$ & $170(6.2)^{*}$ & $168.8(5.8)$ \\
Weight (kg) & $60.5(9.2)$ & $75(12.9)^{\dagger}$ & $62.3(8.4)$ & $76.3(14.6)^{\ddagger}$ \\
Weight at 20 years (kg) & $56.1(5.7)$ & $70.6(10.4)^{\dagger}$ & $58.9(5.5)^{\star}$ & $72.3(10.1)^{\ddagger}$ \\
BMI at 20 years (kg/m $\left.{ }^{\dagger}\right)$ & $20.1(1.5)$ & $25.6(1.3)^{\dagger}$ & $20.4(1.3)$ & $25.4(3.3)^{\ddagger}$ \\
Birth weight (g) & $110.5(405.8)$ & $3098.3(443.3)$ & $3775.2(431.5)^{*}$ & $3837.7(361.6)^{\ddagger}$ \\
Office blood pressure & $n=160$ & $n=136$ & $n=128$ & $n=148$ \\
SBP (mmHg) & $103.4(8.3)$ & $107.2(9.3)^{\dagger}$ & $102.8(11.5)$ & $105.7(9.4)$ \\
DBP (mmHg) & $65[61-71]$ & $68[63-79]^{\dagger}$ & $65[60-69]$ & $66[62-71]$ \\
Pulse wave measurements & $n=158$ & $n=133$ & $n=127$ & $n=145$ \\
Pulse wave velocity (m/s) & $6.3(0.9)$ & $6.2(0.9)$ & $6.9(1.06)$ & $6.2(0.9)$ \\
Augmentation pressure index & $2.8(12.8)$ & $1.8(11.6)$ & $-0.8(11.7)$ & $0.3(11)$ \\
Central SBP (mmHg) & $89[85-95]$ & $92[86-97]^{\dagger}$ & $89[83-94]$ & $91[86-96]$ \\
Central DBP (mmHg) & $65[62-70]$ & $68[64-72]^{\dagger}$ & $65[60-69]$ & $67[62-72]$ \\
ABPM (mmHg) & $n=23$ & $n=28$ & $n=24$ & $n=29$ \\
SBP day & $114(7.9)$ & $118(9.5)$ & $114.9(10.9)$ & $117.6(9.2)$ \\
SBP night & $99.4(8.2)$ & $102.4(10.1)$ & $101.8(11.5)$ & $103.7(9.6)$ \\
DBP day & $65.5(7.3)$ & $68.4(6.9)$ & $65.5(7.9)$ & $68(7.1)$ \\
DBP night & $53.6(5.8)$ & $56(7.2)$ & $53.9(8.4)$ & $55.8(7.7)$ \\
PP day & $48.5(5.2)$ & $49.5(4.6)$ & $49.4(5.1)$ & $48(5.1)$ \\
PP night & $45.8(5.3)$ & $46.4(5.1)$ & $49.6(5.5)$ & $47.9(5.7)$ \\
\hline
\end{tabular}

Data presented as mean $( \pm \mathrm{SD})$ or median [25th-75th percentile] for women was divided into four groups. Low BWz/Low BMI20 (reference group): BWz $\leq 0$ and BMI $\leq$ median. Low BWz/High BMI20: BWz $\leq 0$ and BMI > median. High BWz/Low BMI20: $\mathrm{BWz}>0$ and BMI $\leq$ median. High BWz/High BMI20: $\mathrm{BWz}>0$ and BMI >

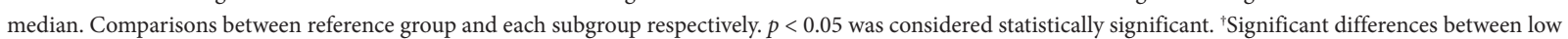
BWz/low BMI20 and low BWz/high BMI20. *Significant differences between low BWz/low BMI20 and high BWz/low BMI20. ${ }^{*}$ Significant differences between low BWz/ low BMI20 and high BWz/high BMI20. $n$, number of subjects. 
Table 5 Odds ratio for elevated blood pressures and higher Pulse Wave Velocity (PWV) as well as Augmentation Index (Aix) above the 75th percentile in the reference group (low BWz/low BMI20). The mismatch category is indicated in bold italic

\begin{tabular}{|c|c|c|c|c|c|c|}
\hline \multirow{2}{*}{ Variables } & \multicolumn{2}{|c|}{$\begin{array}{c}\text { Low BWz/ } \\
\text { High BMI20 }\end{array}$} & \multicolumn{2}{|c|}{$\begin{array}{l}\text { High BWz/ } \\
\text { Low BMI20 }\end{array}$} & \multicolumn{2}{|c|}{$\begin{array}{c}\text { High BWz/ } \\
\text { High BMI20 }\end{array}$} \\
\hline & OR & 95\% CI & OR & 95\% CI & OR & 95\% CI \\
\hline \multicolumn{7}{|c|}{$\mathrm{SBP}(\mathrm{mmHg})$} \\
\hline All & $2.78^{*}$ & $1.94-3.98$ & $0.55^{*}$ & $0.35-0.86$ & $1.92^{*}$ & $1.33-2.78$ \\
\hline Men & $2.31^{*}$ & $1.36-3.93$ & 0.87 & $0.46-1.62$ & 1.72 & $0.98-3.02$ \\
\hline Women & $1.87^{*}$ & $1.12-3.13$ & 0.79 & $0.44-1.41$ & $1.7^{*}$ & $1.02-2.82$ \\
\hline \multicolumn{7}{|c|}{$\mathrm{DBP}(\mathrm{mmHg})$} \\
\hline All & 1.18 & $0.79-1.74$ & $0.5^{*}$ & $0.31-0.8$ & 1.06 & $0.71-1.59$ \\
\hline Men & 1.51 & $0.87-2.61$ & 0.58 & $0.29-1.14$ & 1.33 & $0.75-2.37$ \\
\hline Women & 1.59 & $0.94-2.69$ & 0.54 & $0.29-1.03$ & 1.02 & $0.59-1.75$ \\
\hline \multicolumn{7}{|l|}{$\mathrm{PWV}(\mathrm{m} / \mathrm{s})$} \\
\hline All & 1.13 & $0.78-1.64$ & 1.02 & $0.69-1.51$ & 1.24 & $0.85-1.8$ \\
\hline Men & 1.03 & $0.6-1.76$ & 0.96 & $0.54-1.71$ & 1.48 & $0.86-2.53$ \\
\hline Women & 1.43 & $0.86-2.37$ & 1.52 & $0.91-2.52$ & 0.86 & $0.51-1.45$ \\
\hline \multicolumn{7}{|c|}{ Augmentation index } \\
\hline All & 0.72 & $0.48-1.08$ & 0.67 & $0.44-1.04$ & 0.7 & $0.46-1.06$ \\
\hline Men & 1.1 & $0.62-1.97$ & 0.58 & $0.29-1.17$ & 1.12 & $0.61-2.05$ \\
\hline Women & 0.91 & $0.52-1.58$ & 0.57 & $0.31-1.05$ & 0.55 & $0.3-1$ \\
\hline \multicolumn{7}{|c|}{ Central SBP (mmHg) } \\
\hline All & $2^{*}$ & $1.38-2.91$ & $0.38^{*}$ & $0.23-0.64$ & 1.38 & $0.94-2.04$ \\
\hline Men & $1.79^{*}$ & $1.02-3.13$ & 0.5 & $0.24-1.05$ & 1.49 & $0.82-2.69$ \\
\hline Women & 0.75 & $0.41-1.37$ & 1.58 & $0.93-2.69$ & 1.17 & $0.68-2.01$ \\
\hline \multicolumn{7}{|c|}{ Central DBP (mmHg) } \\
\hline All & 1.2 & $0.81-1.76$ & $0.52^{*}$ & $0.33-0.82$ & 1.25 & $0.85-1.85$ \\
\hline Men & 1.4 & $0.8-2.42$ & 0.55 & $0.27-1.08$ & 1.43 & $0.81-2.53$ \\
\hline Women & 0.86 & $0.48-1.53$ & $1.8^{*}$ & $1.07-3.03$ & 1.54 & $0.92-2.58$ \\
\hline
\end{tabular}

Confidence interval calculated for 95th percentile. Odds ratio was calculated in relation to the 75th percentile in the reference group (low BWz/low BMI20) as cutoff for each variable. Confidence intervals and $p$-values were calculated. "Statistically significant odds ratio. $p<0.05$ was considered statistically significant.

associated with Aix $(\beta=-0.087 ; p=0.003)$ adjusted for sex, age and BMI at examination, SBP, DBP and heart rate. Furthermore, $\mathrm{BWz}$ was inversely associated with $\operatorname{bDBP}(\beta=-0.074 ; p=0.011)$, $\operatorname{cSBP}(\beta=-0.063 ; p=0.033)$ and $\operatorname{cDBP}(\beta=-0.083 ; p=0.005)$, all associations adjusted for age at examination.

\section{DISCUSSION}

Birth weight is determined in part by the genetic growth potential but is also affected by gestational age, prenatal conditions in the uterus and the placental function. During fetal growth, adaptation and genetic programming take place according to the conditions in the prenatal environment, later influenced by the post-natal environment (nutrition). We hypothesized that a phenotypic mismatch exists between prenatal factors and the post-natal environment in some individuals, contributing to changes in central and peripheral hemodynamics in young adults.

The main result was that participants born with low birth weight and with a higher attained adult BMI during the two first decades of life (the mismatch phenotype), showed significantly higher bSBP/ bDBP, cSBP, night-time ambulatory SBP and pulse pressure, compared to the reference group. This finding contrasts to the expected observations in subjects with isolated systolic hypertension related to obesity and metabolic abnormalities, in general characterized by normal central blood pressure and bDBP, but with elevated bSBP [28]. Although, in spite of very few hypertensive participants in "high BWz/high BMI20", this more typical picture was observed with higher bSBP and ambulatory night-time SBP and day- and night-time PP. Furthermore, there was a higher odds ratio of elevated bSBP in "high BWz/high BMI20" (OR 1.98), but with even higher odds in the mismatch category (2.78). Also, the mismatch group was at significantly higher risk of elevated cSBP (OR 2.0), but when stratified for sex this was only true for men (OR 1.79).

Our data support our hypothesis of an independent mismatch phenotype, implying that participants with lower birth weight and higher attained adult BMI are not only at higher risk of elevated peripheral and CSBP, but may also be viewed as a separate phenotype from isolated systolic hypertension associated with higher adult BMI.

Low birth weight by itself is associated with an increased risk of hypertension $[2,4,5]$. This is in line with the finding of higher bDBP, cSBP/cDBP, as well as higher Aix, in our reference group ("low BWz/low BMI20") compared to the subgroup "high BWz/ low BMI20." Furthermore, this subgroup was at lower risk (lower odds ratio) of elevated $\mathrm{bSBP} / \mathrm{bDBP}, \mathrm{cSBP} / \mathrm{cDBP}$ than the reference group. Also, in line with previous data from $\mathrm{MOS}, \mathrm{BWz}$ was inversely associated with Aix and bDBP, as well as with cSBP/cDBP [22]. Clearly, lower birth weight is predictive of elevated blood pressure, brachial as well as central blood pressure, in young adults. As the mismatch group exhibits higher $\mathrm{cSBP} / \mathrm{cDBP}$ compared to the reference group it is suggested that mismatch is also predictive of elevated central blood pressure. Whether this should be considered as an additive effect modifier of low birth weight or as an independent effect of mismatch is unclear.

When stratified for sex, the mismatch group showed significantly higher SBP/DBP and $\mathrm{CSBP} / \mathrm{cDBP}$ in both men and women compared to reference. No significant differences were seen between the group "high BWz/high BMI20" and reference, irrespective of sex. Interestingly, stratifying for sex seems to strengthen our hypothesis of a mismatch phenotype, as the explanatory effect of higher BMI itself seems to weaken.

Further, in men but not in women, bDBP, cSBP/cDBP and Aix was lower in "high BWz/low BMI20" in comparison with reference. Our findings suggest that the effect of low birth weight may be more evident in men, although not true for mismatch group. It is known, that women during their fertile years are less prone to develop hypertension compared to men [29], which may explain the sex difference according to the programming effect of birth weight. However, it seems contradictory that the mismatch group shows higher blood pressure regardless of gender, possibly indicating different pathophysiological pathways behind the elevated blood pressure influenced by low birth weight versus mismatch.

Previous mismatch studies have shown elevated office SBP in young adults characterized by a history of low birth weight and later APG (catch-up growth) [11,18-20]. Similarly to our findings, also elevated DBP in young adults following APG has been reported $[18,19]$. In a British cohort with 346 participants, examined at 22-years of age, a rapid weight gain (catch-up) between 1 and 5 years of age was associated with higher adult blood pressure, in particular for SBP. Following adjustment for adult BMI, the programming effect of rapid weight gain even increased [18]. 
In a similar study of 679 subjects, early rapid catch-up growth during the first 5 months of life was associated with higher SBP and DBP at 25-years of age, but only SBP was associated with later rapid growth (between 9 months and 5 years) [19]. In a cohort of 5198 participants from northern Finland, immediate post-natal growth was associated with adult blood pressure levels as mediated by growth later in life, but also birth weight by itself was inversely associated with adult SBP [20], similar to findings from the MOS cohort. In 243 young adults aged 18-24, post-natal growth, but not low birth weight alone, was associated with adult SBP [11]. In pediatric populations, participants with low birth weight followed by rapid post-natal weight gain were at higher risk of hypertension and obesity, but also higher cf-PWV and $b S B P / b D B P$ were reported $[12,30,31]$. In summary, our finding of higher $\mathrm{bSBP} / \mathrm{bDBP}$ in the mismatch group are in line with previous studies, although conflicting results has been reported regarding office DBP.

Central blood pressure is considered a better predictor of future cardiovascular risk than office blood pressure and is more closely related to target organ damage [32]. However, few studies have examined central hemodynamics (i.e., central blood pressures, cf-PWV, and Aix) in relation to birth weight and the mismatch concept, especially in adults. Similar to our results, higher cSBP was reported in obese adults born with low birth weight [33], although we additionally reported elevated CSBP in those born with both low birth weight and attained higher adult weight. Furthermore, no significant differences in cf-PWV were observed between any of our sub-groups and the reference group, neither in men nor in women. Previously, cf-PWV in relation to mismatch has been poorly studied, and conflicting findings were reported [12,34]. In children 8-11 years old, born with lower birth weight and after shorter gestation followed by accelerated post-natal growth, a higher cf-PWV and SBP was noticed [12]. However, in adolescents 16-19 years, accelerated post-natal growth following low birth weight was associated with elevated SBP, but not cf-PWV [34]. Regarding Aix, an estimate of aortic reflection waves [35], we observed lower Aix in "high BWz/low BMI20" and "high BWz/high BMI20" compared to "low BWz/low BMI20 (reference), implying that lower birth weight associates with higher Aix. Similar to our findings, higher adult Aix was reported in men born with low birth weight [4], but not in obese adults born with low birth weight [33]. Our results suggest, in general, an unfavorable genetic programming effect of lower birth weight on elevated adult cSBP, when a higher attained BMI20 tends to add to the risk.

Regarding central arterial stiffness, no differences in cf-PWV or Aix were observed in comparison between mismatch group and the reference group. This finding was somewhat surprising, considering that central aortic pressure is closely related to left ventricular volume and central aortic stiffness $[35,36]$. Although Aix has been suggested a more sensitive marker of arterial stiffness than cf-PWV in younger subjects [37], the absence of subgroup differences in cf-PWV should be interpreted with caution, due to the relatively low mean age of the MOS participants. Furthermore, in a previous MOS publication, birth weight was reported to be positively associated with cf-PWV and inversely associated with Aix [22]. As high maternal BMI, often associated with hyperglycemia, is a risk condition for macrosomia, it was suggested that the positive association between birth weight and cf-PWV could possibly be explained by a secular trend of rising BMI in pregnant women in Sweden [38], observations also reported from India [39]. Likewise, Koivistoinen et al. [40] reported that the metabolic syndrome in children at the age of 9-11 years was predictive of elevated PWV after a 21-year follow-up.

In summary, we have no unambiguous explanation for why only central blood pressure was elevated, but not arterial stiffness based on cf-PWV in the mismatch group. Further studies are wanted, the influences of early life factors on ventricular-arterial coupling [41] could for example be one possible future research area, including the effects of changes in vascular impedance and imaging studies of cardiac function.

This observational study cannot prove causality; and therefore caution should be applied when interpreting the results. A weakness of this study is the simplified model of mismatch, with comparison of four subgroups constituted by BWz and BMI20. In comparison to other studies, we unfortunately lack recordings from postnatal growth patterns during the first few years of life. However, as an attempt to overcome this weakness, we used self-reported body weight at 20 years, or actual weight for those under 20 years, to calculate BMI when creating subgroups.

A considerable strength of our analyses is that we included highly accurate birth data from the national MBR. In addition, we also used specific data derived from Swedish birth cohorts [42] to calculate BWz, adjusted for sex and gestational age, thereby reducing confounding from prematurity. Furthermore, we have been able to include a relatively high number of participants in comparison to other smaller studies. Additionally, we are using gold-standard methods for PWV and central pulse wave recordings, adding data on central blood pressure. To the best of our knowledge, this is the first study in young adults to examine central blood pressures in relation to the mismatch concept.

The concept of a mismatch phenotype presupposes a complex multifactorial etiology and there is still a lack of knowledge. Of particular interest for future research would be the inclusion of maternal primary care and child health care medical records to get further data of maternal exposures as well as pediatric growth charts. Also genetic factors influencing birth weight, weight trajectories and blood pressure regulation should be more studied [43]. The role of prolonged breastfeeding [44] and a balanced diet [45] to avoid rapid catch-up growth should be more studied as a way to prevent adult hypertension.

\section{CONCLUSION}

Lower birth weight associates with higher brachial and central blood pressure as well as Aix. Lower birth weight, in combination with a higher attained BMI in young adult life (the mismatch phenotype) associates with even higher brachial and central blood pressure, in a similar way for both men and women. We suggest the existence of a mismatch phenotype for influencing central hemodynamics based on the additive programming effect of weight gain following low birth weight. Therefore, children born with low birth weight should be protected from exaggerated catch-up growth to reduce their risk of adult hypertension, obesity, and adverse central hemodynamics. Prolonged breastfeeding could be one possible alternative to achieve such a more balanced post-natal growth pattern. 


\section{CONFLICTS OF INTEREST}

The authors declare they have no conflicts of interest.

\section{AUTHORS' CONTRIBUTION}

JS and PMN provided the idea for the analyses that were done by JS. JS drafted the first manuscript that was later read and revised by all authors (JS, SS, PMN). PMN is the guarantor of the study.

\section{ACKNOWLEDGMENTS}

This study was funded by the Research Council of Sweden, the Heart- and Lung Foundation and the Region Skane County Council (ALF grants) to P.M.N.

\section{SUPPLEMENTARY MATERIALS}

Supplementary data related to this article can be found at https:// doi.org/10.2991/artres.k.210215.001.

\section{REFERENCES}

[1] Lawlor DA, Ronalds G, Clark H, Smith GD, Leon DA. Birth weight is inversely associated with incident coronary heart disease and stroke among individuals born in the 1950s: findings from the Aberdeen Children of the 1950s prospective cohort study. Circulation 2005;112:1414-18.

[2] te Velde SJ, Ferreira I, Twisk JWR, Stehouwer CDA, van Mechelen W, Kemper HCG, et al. Birthweight and arterial stiffness and blood pressure in adulthood-results from the Amsterdam Growth and Health Longitudinal Study. Int J Epidemiol 2004;33:154-61.

[3] Mzayek F, Sherwin R, Hughes J, Hassig S, Srinivasan S, Chen W, et al. The association of birth weight with arterial stiffness at mid-adulthood: the Bogalusa Heart Study. J Epidemiol Community Health 2009;63:729-33.

[4] Miles KL, McDonnell BJ, Maki-Petaja KM, Yasmin, Cockcroft JR, Wilkinson IB, et al. The impact of birth weight on blood pressure and arterial stiffness in later life: the Enigma Study. J Hypertens 2011;29:2324-31.

[5] Tan M, Cai L, Ma J, Jing J, Ma Y, Chen Y. The association of gestational age and birth weight with blood pressure among children: a Chinese national study. J Hum Hypertens 2018;32:651-9.

[6] Paneth N, Susser M. Early origin of coronary heart disease (the "Barker hypothesis"). BMJ 1995;310:411-12.

[7] Hanson MA, Gluckman PD. Developmental origins of health and disease: new insights. Basic Clin Pharmacol Toxicol 2008;102:90-3.

[8] Huang L, Yang S, Yang F, Xiong F. A prospective study about physical growth of children from birth to 2 years old born full-term small-for-gestational-age. J Paediatr Child Health 2019;55:199-204.

[9] Larsson A, Ottosson P, Törnqvist C, Olhager E. Body composition and growth in full-term small for gestational age and large for gestational age Swedish infants assessed with air displacement plethysmography at birth and at 3-4 months of age. PLoS One 2019;14:e0207978.
[10] Kelishadi R, Haghdoost AA, Jamshidi F, Aliramezany M, Moosazadeh M. Low birthweight or rapid catch-up growth: which is more associated with cardiovascular disease and its risk factors in later life? A systematic review and cryptanalysis. Paediatr Int Child Health 2015;35:110-23.

[11] Leunissen RWJ, Kerkhof GF, Stijnen T, Hokken-Koelega ACS. Effect of birth size and catch-up growth on adult blood pressure and carotid intima-media thickness. Horm Res Paediatr 2012; 77:394-401.

[12] Mora-Urda AI, Acevedo P, Montero López MP. Relationship between prenatal and postnatal conditions and accelerated postnatal growth. Impact on the rigidity of the arterial wall and obesity in childhood. J Dev Orig Health Dis 2019;10:436-46.

[13] Lei X, Chen Y, Ye J, Ouyang F, Jiang F, Zhang J. The optimal postnatal growth trajectory for term small for gestational age babies: a prospective cohort study. J Pediatr 2015;166:54.e3-8.e3.

[14] Darendeliler F, Bas F, Bundak R, Coban A, Disci R, Sancakli O, et al. Elevated ghrelin levels in preterm born children during prepubertal ages and relationship with catch-up growth. Eur J Endocrinol 2008;159:555-60.

[15] Vianna CA, Horta BL, Gigante DP, de Barros FCLF. Pulse wave velocity at early adulthood: breastfeeding and nutrition during pregnancy and childhood. PLoS One 2016;11:e0152501.

[16] Fabricius Bjerre S, Jensen RB, Færch K, Larsen T, Mølgaard C, Michaelsen KF, et al. Impact of birth weight and early infant weight gain on insulin resistance and associated cardiovascular risk factors in adolescence. PLoS One 2011;6:e20595.

[17] Dennison BA, Edmunds LS, Stratton HH, Pruzek RM. Rapid infant weight gain predicts childhood overweight. Obesity (Silver Spring) 2006;14:491-9.

[18] Law CM, Shiell AW, Newsome CA, Syddall HE, Shinebourne EA, Fayers PM, et al. Fetal, infant, and childhood growth and adult blood pressure: a longitudinal study from birth to 22 years of age. Circulation 2002;105:1088-92.

[19] Ben-Shlomo Y, McCarthy A, Hughes R, Tilling K, Davies D, Smith GD. Immediate postnatal growth is associated with blood pressure in young adulthood: the Barry Caerphilly Growth Study. Hypertension 2008;52:638-44.

[20] Kaakinen M, Sovio U, Hartikainen AL, Pouta A, Savolainen MJ, Herzig $\mathrm{KH}$, et al. Life course structural equation model of the effects of prenatal and postnatal growth on adult blood pressure. J Epidemiol Community Health 2014;68:1161-7.

[21] Leon DA, Koupilova I, Lithell HO, Berglund L, Mohsen R, Vagero D, et al. Failure to realise growth potential in utero and adult obesity in relation to blood pressure in 50 year old Swedish men. BMJ 1996;312:401-6.

[22] Sperling J, Nilsson PM. Does early life programming influence arterial stiffness and central hemodynamics in adulthood? J Hypertens 2020;38:481-8.

[23] Brunkwall L, Jönsson D, Ericson U, Hellstrand S, Kennbäck C, Östling G, et al. The Malmö Offspring Study (MOS): design, methods and first results. Eur J Epidemiol 2021;36:103-16.

[24] Rosberg EP, Nilsson PM. Family patterns of arterial stiffness across three generations in the Malmö Offspring Study. J Hypertens 2020;38:474-80.

[25] Guo QH, Muhammad IF, Borné Y, Sheng CS, Persson M, Wang JG, et al. Difference in the risk profiles of carotid-femoral pulse wave velocity: results from two community-based studies in China and Sweden. J Hum Hypertens 2020;34:207-13. 
[26] Niklasson A, Ericson A, Fryer JG, Karlberg J, Lawrence C, Karlberg P. An update of the swedish reference standards for weight, length and head circumference at birth for given gestational age (1977-1981). Acta Paediatr Scand 1991;80:756-62.

[27] Bland JM, Altman DG. Statistics notes. The odds ratio. BMJ 2000;320:1468.

[28] Isolated systolic hypertension in the young: a position paper endorsed by the European Society of Hypertension. J Hypertens 2018;36:2120.

[29] Ashraf MS, Vongpatanasin W. Estrogen and hypertension. Curr Hypertens Rep 2006;8:368-76.

[30] Cheung YB, Low L, Osmond C, Barker D, Karlberg J. Fetal growth and early postnatal growth are related to blood pressure in adults. Hypertension 2000;36:795-800.

[31] Marinkovic T, Toemen L, Kruithof CJ, Reiss I, van Osch-Gevers L, Hofman A, et al. Early infant growth velocity patterns and cardiovascular and metabolic outcomes in childhood. J Pediatr 2017;186:57.e4-63.e4.

[32] Cziraki A, Husznai R, Nemeth A, Lenkey Z, Bocskei R, Gaszner B, et al. The investigation of central arterial stifness and central blood pressure parameters in various cardiovascular diseases. Eur Heart J 2018;39:1546.

[33] Lurbe E, Carvajal E, Torro I, Aguilar F, Alvarez J, Redon J. Influence of concurrent obesity and low birth weight on blood pressure phenotype in youth. Hypertension 2009;53:912-17.

[34] Salvi P, Revera M, Joly L, Reusz G, Iaia M, Benkhedda S, et al. Role of birth weight and postnatal growth on pulse wave velocity in teenagers. J Adolesc Health 2012;51:373-9.

[35] Laurent S, Cockcroft J, Van Bortel L, Boutouyrie P, Giannattasio C, Hayoz D, et al. Expert consensus document on arterial stiffness: methodological issues and clinical applications. Eur Heart J 2006;27:2588-605.

[36] Roman MJ, Okin PM, Kizer JR, Lee ET, Howard BV, Devereux RB. Relations of central and brachial blood pressure to left ventricular hypertrophy and geometry: the Strong Heart Study. J Hypertens 2010;28:384-8.

[37] McEniery CM, Yasmin, Hall IR, Qasem A, Wilkinson IB, Cockcroft JR. Normal vascular aging: differential effects on wave reflection and aortic pulse wave velocity: the AngloCardiff Collaborative Trial (ACCT). J Am Coll Cardiol 2005; 46:1753-60.

[38] Socialstyrelsen. The National Board of Health and Welfare. Statistics on Pregnancies, Deliveries and Newborn Infants 2016. 2018. Available from: https://www.socialstyrelsen.se/globalassets/ sharepoint-dokument/artikelkatalog/statistik/2018-1-7.pdf.

[39] Yajnik CS, Yajnik PC. Fetal adiposity epidemic in the modern world: a thrifty phenotype aggravated by maternal obesity and diabetes. Am J Clin Nutr 2020;112:8-10.

[40] Koivistoinen T, Hutri-Kähönen N, Juonala M, Aatola H, Kööbi T, Lehtimäki T, et al. Metabolic syndrome in childhood and increased arterial stiffness in adulthood - The Cardiovascular Risk in Young Finns Study. Ann Med 2011;43:312-19.

[41] Ikonomidis I, Aboyans V, Blacher J, Brodmann M, Brutsaert DL, Chirinos JA, et al. The role of ventricular-arterial coupling in cardiac disease and heart failure: assessment, clinical implications and therapeutic interventions. A consensus document of the European Society of Cardiology Working Group on Aorta \& Peripheral Vascular Diseases, European Association of Cardiovascular Imaging, and Heart Failure Association. Eur J Heart Fail 2019; 21:402-24.

[42] Källén B, Källén K. The Swedish Medical Birth Register - a summary of content and quality. Stockholm: Socialstyrelsen; 2003.

[43] Warrington NM, Beaumont RN, Horikoshi M, Day FR, Helgeland $\varnothing$, Laurin C, et al. Maternal and fetal genetic effects on birth weight and their relevance to cardio-metabolic risk factors. Nat Genet 2019;51:804-14.

[44] de Beer M, Vrijkotte TGM, Fall CHD, van Eijsden M, Osmond C, Gemke RJBJ. Associations of infant feeding and timing of weight gain and linear growth during early life with childhood blood pressure: findings from a prospective population based cohort study. PLoS One 2016;11:e0166281.

[45] Intapad S, Dasinger JH, Johnson JM, Brown AD, Ojeda NB, Alexander BT. Male and female intrauterine growth-restricted offspring differ in blood pressure, renal function, and glucose homeostasis responses to a postnatal diet high in fat and sugar. Hypertension 2019;73:620-9. 